\title{
Response to: Letter to the Editor Concerning "A fully dynamic multi-compartmental poroelastic system: Application to aqueductal stenosis"
}

\author{
John Vardakis ${ }^{1}$, Dean Chou ${ }^{2}$, Liwei Guo ${ }^{1}$, Brett J. Tully ${ }^{3}$ and Yiannis Ventikos ${ }^{1}$ \\ 1Department of Mechanical Engineering, University College London, UK, y.ventikos@ucl.ac.uk \\ ${ }^{2}$ Institute of Biomedical Engineering \& Department of Engineering Science, University of Oxford, UK \\ ${ }^{3}$ Children's Medical Research Institute and School of Medical Sciences, Sydney Medical School, The University of Sydney, \\ Westmead, Australia
}

We would like to thank Dr. A. Mehrabian and Professor Y. Abousleiman for their interest regarding our paper. In their correspondence with the editor, they highlighted three main areas that require further clarification with regards to the publication by (Chou et al., 2016). These are: (i) the omission of cross-porosity storage effects; (ii) the constitutive relation allied to fluid content; and (iii) concerning the constraints on the numerical values for the Biot-Willis effective stress coefficient. Our aim in this letter is to respond to the aforementioned points raised by Mehrabian and Abousleiman, in addition to providing some further insight into the rationale behind the assumptions adopted in (Chou et al., 2016). For clarity, we provide the numbered list of equations given by Mehrabian and Abousleiman that are relevant to the discussion:

$$
\begin{aligned}
& S_{\varepsilon}^{X Y}=\left.\frac{\partial \zeta_{X}}{\partial p_{Y}}\right|_{\varepsilon=p_{Z}=0} \quad,(Z \neq Y) \\
& S_{\varepsilon}^{a} \dot{p}_{a}+S_{\varepsilon}^{a c} \dot{p}_{c}+S_{\varepsilon}^{a e} \dot{p}_{e}+S_{\varepsilon}^{a v} \dot{p}_{v}+\alpha^{a} \dot{\varepsilon}-\kappa^{a} \nabla^{2} p_{a}=\sum_{X=c, e, v} \omega_{a X}\left(p_{X}-p_{a}\right) \\
& \zeta_{a}=\alpha^{a} \varepsilon_{i i}+S_{\varepsilon}^{a} p_{a}+S_{\varepsilon}^{a c} p_{c}+S_{\varepsilon}^{a e} p_{e}+S_{\varepsilon}^{a v} p_{v} \\
& \left(\begin{array}{c}
\sigma \\
\zeta_{a} \\
\zeta_{c} \\
\zeta_{e} \\
\zeta_{v}
\end{array}\right)=\left(\begin{array}{ccccc}
K & -\alpha^{a} & -\alpha^{c} & -\alpha^{e} & -\alpha^{v} \\
\alpha^{a} & S_{\varepsilon}^{a} & S_{\varepsilon}^{a c} & S_{\varepsilon}^{a e} & S_{\varepsilon}^{a v} \\
\alpha^{c} & S_{\varepsilon}^{a c} & S_{\varepsilon}^{c} & S_{\varepsilon}^{c e} & S_{\varepsilon}^{c v} \\
\alpha^{e} & S_{\varepsilon}^{a e} & S_{\varepsilon}^{c e} & S_{\varepsilon}^{e} & S_{\varepsilon}^{e v} \\
\alpha^{v} & S_{\varepsilon}^{a v} & S_{\varepsilon}^{c v} & S_{\varepsilon}^{e v} & S_{\varepsilon}^{v}
\end{array}\right) \cdot\left(\begin{array}{c}
\varepsilon \\
p_{a} \\
p_{c} \\
p_{e} \\
p_{v}
\end{array}\right) \\
& \left(\begin{array}{l}
\sigma \\
\zeta
\end{array}\right)=\left(\begin{array}{cc}
K & -\sum_{X=a, e, c, v} \alpha^{X} \\
\sum_{X=a, e, c, v} \alpha^{X} & \sum_{X, Y=a, e, c, v} S_{\varepsilon}^{X Y}
\end{array}\right) \cdot\left(\begin{array}{l}
\varepsilon \\
p
\end{array}\right) \\
& \alpha=\left.\frac{\partial \zeta}{\partial \varepsilon}\right|_{p=0}=-\left.\frac{\partial \sigma}{\partial p}\right|_{\varepsilon=0}=\sum_{X=a, e, c, v} \alpha^{X} \\
& \phi \leq \alpha \leq 1
\end{aligned}
$$

We commence the discussion by outlining the need to perceive the multiple-network poroelasticity model outlined in (Chou et al., 2016) from a physiological point of view. Defining a subject-specific formulation for the MPET system is currently in its nascent stages of development. Some of the aspects outlined by the 
aforementioned authors are a reflection of this evolution. The paper extends on the quasi-steady formulation adopted in previous work (Tully and Ventikos, 2010, Vardakis et al., 2016, Vardakis et al., 2014), by exploring the ability of the MPET template to cope with additional inertial terms.

There is great uncertainty regarding the properties and even the nature of the base constitutive equation, regarding the parenchyma solid matrix, in multiphasic models or any other paradigm. Parenchyma does not fall within the well-defined categories of fractured media (fractured, totally-fractured or partially fractured). Therefore, given this uncertainty, we have opted for a casting of the equations that is as simple, mechanically, as possible, while attempting to maintain and incorporate physiology elements and mechanisms that are important. We believe it is more useful (at this stage) to outline the definition of the perfused parenchymal tissue using a composite Barenblatt diffusion-type system (quadruple-diffusion model) (Barenblatt et al., 1960), as this harnesses the classical description of diffusion in the medium. In this way, one may introduce pressure dependent variations due to the four compartments through a sufficiently large representative elementary volume (REV). This is important since we need to be able to account for the arterial and venous compartments, for the reasons described below. There are numerous sources of Barenblatt-Biot systems representing double diffusion in poroelastic media that have been described in the literature (Aifantis, 1980, Beskos and Aifantis, 1986, Wilson and Aifantis, 1982, Showalter, 2000, Showalter, 2002, Showalter and Momken, 2002, Khaled et al., 1984), and these form a basis to develop a framework in which we can initiate a formal discussion on the intricate nature of CSF/ISF transport. We note that a generic representation of the cerebral parenchyma is adequately defined by using just two compartments in the MPET system, namely the capillary and CSF/ISF network. In this way, established fluid exchange mechanisms of interest (such as the blood-brain barrier, which is a diffusion barrier essential for the normal function of the central nervous system) would occur between these two distinct compartments. In the MPET models that have used four compartments (Chou et al., 2016, Vardakis et al., 2016, Vardakis et al., 2014, Tully and Ventikos, 2010), we make the assumption that the proximity between this dual-compartment (capillary-CSF/ISF) and an artery or a vein is small enough for these two larger vessels to be incorporated within a REV of parenchymal tissue. This is an important assumption, as the conceptualisation of the recently discovered glymphatic pathway (Iliff et al., 2012) must be catered for in any ensuing discussion revolving around CSF transport.

The physiology, velocity scales and time scales of relevance in this class of problems indicate that compressibility effects have minimal influence, if any. The adopted formulation essentially excludes wave motion in the fluid phases by virtue of the fact that the fluid acceleration relative to the solid matrix (including the convective acceleration terms) is omitted in the formulation of equations 9a-e from from (Chou et al., 2016). This is clearly stated in the description of the formulation. We revisit these equations by defining them below:

$$
\begin{aligned}
& {\left[G \nabla^{2} \mathbf{u}+\left(\frac{G}{1-2 v}\right) \nabla(\nabla \cdot \mathbf{u})-\alpha^{a} \nabla p^{a}-\alpha^{c} \nabla p^{c}-\alpha^{e} \nabla p^{e}-\alpha^{v} \nabla p^{v}\right]=\rho \frac{\partial^{2} \mathbf{u}}{\partial t^{2}}} \\
& \frac{\partial}{\partial t}\left(S_{\varepsilon}^{a} p^{a}+\alpha^{a} \nabla \cdot \mathbf{u}\right)-\left[\frac{k_{a}}{\mu_{a}} \nabla^{2} p^{a}+\left|\hat{s}_{a \rightarrow c}\right|\right]=\frac{k_{a} \rho^{a}}{\mu_{a}} \frac{\partial^{2}}{\partial t^{2}}(\nabla \cdot \mathbf{u}) \\
& \frac{\partial}{\partial t}\left(S_{\varepsilon}^{c} p^{c}+\alpha^{c} \nabla \cdot \mathbf{u}\right)-\left[\frac{k_{c}}{\mu_{c}} \nabla^{2} p^{c}-\left|\hat{s}_{a \rightarrow c}\right|+\left|\hat{s}_{c \rightarrow e}\right|+\left|\hat{s}_{c \rightarrow v}\right|\right]=\frac{k_{c} \rho^{c}}{\mu_{c}} \frac{\partial^{2}}{\partial t^{2}}(\nabla \cdot \mathbf{u}) \\
& \frac{\partial}{\partial t}\left(S_{\varepsilon}^{e} p^{e}+\alpha^{e} \nabla \cdot \mathbf{u}\right)-\left[\frac{k_{e}}{\mu_{e}} \nabla^{2} p^{e}-\left|\hat{s}_{c \rightarrow e}\right|+\left|\hat{s}_{e \rightarrow v}\right|\right]=\frac{k_{e} \rho^{e}}{\mu_{e}} \frac{\partial^{2}}{\partial t^{2}}(\nabla \cdot \mathbf{u}) \\
& \frac{\partial}{\partial t}\left(S_{\varepsilon}^{v} p^{v}+\alpha^{v} \nabla \cdot \mathbf{u}\right)-\left[\frac{k_{v}}{\mu_{v}} \nabla^{2} p^{v}-\left|\hat{s}_{c \rightarrow v}\right|-\left|\hat{s}_{e \rightarrow v}\right|\right]=\frac{k_{v} \rho^{v}}{\mu_{v}} \frac{\partial^{2}}{\partial t^{2}}(\nabla \cdot \mathbf{u})
\end{aligned}
$$

From equation 8a, the additional inertial term is $\rho \frac{\partial^{2} \mathbf{u}}{\partial t^{2}}$, whilst in equations $8 \mathrm{~b}$-e, it is $\frac{k_{i} \rho^{i}}{\mu_{i}} \frac{\partial^{2}}{\partial t^{2}}(\nabla \cdot \mathbf{u})$, where $i=$ $a, e, c, v$. This is the $\mathbf{u}-p$ formulation presented in (Zienkiewicz and Shiomi, 1984), and the physiologically relevant form was outlined in (Tully and Ventikos, 2010). As stated in (Zienkiewicz and Shiomi, 1984), with the exception of gas flow, storage increase (a measure of the compressibility of the pore and fissure system) is negligible under these conditions. The same authors clearly state it is safe to assume that $S_{\varepsilon}^{i} \rightarrow 0$. Considering that all fluids 
percolating the solid matrix in such a physiological setting are incompressible, it is theoretically valid to assume $S_{\varepsilon}^{i} \rightarrow 0$. For instance, this has already been stipulated by (Miga et al., 2000a, Miga et al., 2000b), who assumed the corresponding CSF/ISF fluid is incompressible $\left(S_{\varepsilon}^{e}=0\right)$. In the assumption of incompressibility, this would also theoretically imply $\alpha^{\mathrm{e}}=1$, and that the Poisson's ratio $v=0.5$, in order for the parenchyma to experience the strongest poroelastic effects (Detournay and Cheng, 1993). In the work conducted by (Li et al., 2013), a value of $S_{\varepsilon}^{e}=0.447 \mu \mathrm{Pa}^{-1}$ was used to conduct a parametric study. In their work, the importance of $S_{\varepsilon}^{e}$ was highlighted (dominant parameter that influences the infusion process). In the work of (Chou et al., 2016), this value was used throughout all compartments for simplicity, and in the absence of valid alternatives for the arterial (a), capillary (c) and venous (v) compartments. This assumption (of equal $S_{\varepsilon}^{i}$, in addition to the crossstorage terms possessing the same value as $S_{\varepsilon}^{i}$ ) has been used by (Khalili, 2003), when considering pore-fluid and fissure-fluid incompressibility. $S_{\varepsilon}^{e}$ could have also been calculated from the values in Table 1 from (Chou et al., 2016), which would have provided $S_{\varepsilon}^{e} \approx 22 \mu \mathrm{Pa}^{-1}$. The resultant variation in ventriculomegaly and CSF/ISF pressure was negligible. Specifically, the relative error (between $S_{\varepsilon}^{e}=0.447 \mu \mathrm{Pa}^{-1}$ and $22 \mu P a^{-1}$ ) for ventricular displacement and ventricular CSF/ISF pore pressure fell from a peak of $0.007 \%$ and $0.003 \%$ at $t=0$ hours, to negligible values after 18 hours of simulation time. We therefore opted to use $S_{\varepsilon}^{e}=0.447 \mu P a^{-1}$, as it was derived under more stringent physiological criteria.

We agree with Mehrabian and Abousleiman in that the constant strain cross-storage effects are required for completeness, however, the usefulness of their inclusion in our model is debatable. As mentioned in (Lewallen and Wang, 1998), neglecting the cross-storage coefficient in a double porosity medium yields a small underestimation in fracture pressure, and a rather pronounced underestimation of the matrix pressure during very early simulation times. (Khalili, 2003) also outlines the influence of neglecting the microscopic coupling effects in a double porosity medium. In the latter, the response of the system during the initial period was deemed to be a function of the microscopic coupling between pore and fissure volumetric deformations. Neglecting the cross-coupling terms clearly resulted in a sudden and discontinuous drop in the fissure fluid pressure, and a discontinuous rise in the fluid pressure in the clay matrix. However, the response for the two final stages of the consolidation response in the fissured medium did not follow this development, as the results show very similar trends between coupled (incorporating cross-coupling terms) and uncoupled responses. These rely on the compressibility contrast and the transfer coefficients scaling the flow between networks (defined by $\omega_{\mathrm{ij}}$ in (Chou et al., 2016)), prior to the consolidation behaviour of the system approaching that of the equivalent single compartment medium. All of the simulations conducted in the four network model are conducted with long time scales in mind (gradual aqueductal stenosis severity).

The additional coupling terms per compartment are also prone to individual scrutiny. To make this clear, we outline the extended version of equation $8 \mathrm{~b}$, which takes into account the proposed cross-coupling storage terms proposed by Mehrabian and Abousleiman:

$$
\frac{\partial}{\partial t}\left(S_{\varepsilon}^{a} p^{a}+\left[S_{\varepsilon}^{a c} p_{c}+S_{\varepsilon}^{a e} p_{e}+S_{\varepsilon}^{a v} p_{v}\right]+\alpha^{a} \nabla \cdot \mathbf{u}\right)-\frac{k_{a}}{\mu_{a}} \nabla^{2} p^{a}+\left|\hat{s}_{a \rightarrow c}\right|=\frac{k_{a} \rho^{a}}{\mu_{a}} \frac{\partial^{2}}{\partial t^{2}}(\nabla \cdot \mathbf{u})
$$

In equation 9 above, the additional terms relating cross-coupling storage effects are given in square brackets. The additional material constants are $S_{\varepsilon}^{a c}, S_{\varepsilon}^{a e}$ and $S_{\varepsilon}^{a v}$. In a similar fashion, for the capillary, CSF/ISF and venous compartment we have the additional constants: $S_{\varepsilon}^{c e}, S_{\varepsilon}^{c v}$ and $S_{\varepsilon}^{v e}$. These can also be identified from the off diagonal terms in equation 4 . The general form of such multiple porosity and multiple permeability systems has been highlighted in recent work by Mehrabian and Abousleiman (Mehrabian and Abousleiman, 2015, Mehrabian and Abousleiman, 2014). From this list, it is evident that $S_{\varepsilon}^{a v}$ has no meaning in our system, as the physiological relevance to this term is questionable: An artery and a vein are not directly interconnected, as it is well established that a capillary network must mediate this fluid communication (unless we consider arteriovenous malformations of course) in parenchymal tissue. A refined approach to account for the storage terms has been made, and it takes into account an extended MPET model that incorporates the glymphatic hypothesis of cerebral waste elimination. As one would expect, this has implications for equations 2 and 3 . Once cross-coupling storage effects are taken into account, this equation is extended to reflect the form given by the aforementioned equations. 
Regarding the Biot-Willis effective stress coefficients, Mehrabian and Abousleiman touch upon an interesting topic. The global Biot-Willis constant should not exceed unity under the constraints of equation 7. The underlying reason why this is the case is a blend of numerical template simplicity and physiological realism necessary at the compartmental level. As discussed by (Levine, 2000, Levine, 1999), blood is incompressible, however it flows through vessels that possess flexible walls, which advises a degree of pseudo-compressibility be apparent in the relevant blood compartment (instantaneous compressibility in response to a mechanical compressive stress). In addition to vessel compressibility, the parenchymal displacement is assumed positive in the radial direction in addition to allowing for ventricle expansion. (Levine, 2000) also makes the case for $\alpha^{\text {blood }}>$ 1 in the proposed dual compartment formulation, where under the influence of a compressive stress, the resulting negative (towards the ependymal surface) parenchymal tissue displacement translates to vessel dilation and reduced cerebroventricular compression. (Wirth and Sobey, 2006) also used this approach in describing the influence this had on idiopathic intracranial hypertension (with respect to varying CSF outflow resistance). The work of (Tully and Ventikos, 2010) proposed a means by which this conceptual framework extended to the CSF/ISF and blood (arterial, capillary and venous) compartments, treating each compartment in its own right (yet still allowing for a degree of communication allocated by the intercompartmental fluxes), and satisfying the special case of incompressibility for all the fluid networks $\left(S_{\varepsilon}^{i}=0, \alpha^{i}=1\right)$. As discussed earlier, the strongest poroelastic effects to be imposed upon the parenchymal tissue are assigned when $v=0.5$, in addition to $S_{\varepsilon}^{i}=0$ and $\alpha^{i}=1$. In the work of (Chou et al., 2016), a widely used value of $v=0.35$ was used instead, as the compressible effects allow for changes in fluid to be sustained with the value of Young's modulus adopted (see (Kaczmarek et al., 1997)). The Biot-Willis coefficient of 0.9955 was used, as it matched the baseline model values of (Li et al., 2013). The global effective Biot-Willis coefficient $(\alpha=3.982$ ) for the quadruple-porosity material defined by equation 7 did not adversely influence ventriculomegaly, as the influence of the intercompartmental flux variations (which in turn are also driven by the boundary conditions of the MPET model) also play an important role. It should be noted that considering the model proposed by (Chou et al., 2016) incorporated the constraint of a rigid skull, and that the outer surface of the brain is tethered to the dura. This implies that the subarachnoid space cannot expand; certainly an assumption that needs further exploring. The cumulative vascular response of the parenchymal tissue is largely expressed by the global effective Biot-Willis coefficient of the quadruple-porosity model. Ventricular enlargement depends on the extent of the compressibility imposed by the intertwined vasculature, in addition to the degree of tethering between dura and cortical surface. In (Chou et al., 2016), these are assumed to be fused. Considering $\sum \alpha^{i}>1$, one would expect to observe an overall parenchymal expansion through a simultaneous reduction in cerebroventricular size, in line with observations from (Levine, 2000, Wirth and Sobey, 2006). However, as the parenchyma is constrained from moving inwards (towards the ventricles) by virtue of the imposed fixed boundary condition at the skull, the ventricles are forced to expand. This intrinsically implies that as each compartment possesses a small degree of compressibility $\left(\alpha^{i}=\right.$ 0.9955), the perfused parenchymal tissue yields a consolidated vasodilatory effect. This is physiologically plausible, and described extensively by (Levine, 2000, Levine, 1999). As described in the latter, although the caliber of the capillaries and veins may be maintained, the arteries may expand, especially under the influence of an isotropic compressive stress or an increase in interstitial fluid pressure (decreased contractile tension of the arterial wall). It is therefore important to be able to accommodate this scenario within the MPET framework, as it is a requirement for modelling personalisation.

We believe that omitting the cross-storage effects and depicting the perfused parenchymal tissue as a Barenblatt-Biot systems is a reasonable simplification and approximation for dealing with the problem in question, especially if we further consider the fact that the boundary conditions of each compartment provide intercompartmental flux variations that impose themselves in a significant way when considering the parenchymal tissue displacement. Subject-specific boundary conditions (such as arterial flow profiles arising from the internal carotid and vertebral arteries) that would drive the fluid exchange of the MPET system are realistically applied at the arterial compartment. Being able to account for the potential differentiation in caliber between blood vessels, in addition to the CSF/ISF space is therefore essential when attempting to personalise the MPET system for clinical requirements. The latter point (i.e. clinical relevance) has invariably been an important consideration of ours when constructing this model and deciding upon the appropriate levels of complexity/simplification. 


\section{Conflict of Interest}

The authors report no conflict of interest with respect to this work.

\section{References}

AIFANTIS, E. C. 1980. On the problem of diffusion in solids. Acta Mechanica, 37, 265-296.

BARENBLATT, G. I., ZHELTOV, I. P. \& KOCHINA, I. N. 1960. Basic concepts in the theory of seepage of homogeneous liquids in fissured rocks [strata]. Journal of Applied Mathematics and Mechanics, 24, 1286-1303.

BESKOS, D. E. \& AIFANTIS, E. C. 1986. On the theory of consolidation with double porosity-II. International Journal of Engineering Science, 24, 1697-1716.

CHOU, D., VARDAKIS, J. C., GUO, L., TULLY, B. J. \& VENTIKOS, Y. 2016. A fully dynamic multi-compartmental poroelastic system: Application to aqueductal stenosis. Journal of Biomechanics, 49, 2306-2312.

DETOURNAY, E. \& CHENG, A. H. D. 1993. Fundamentals of poroelasticity. Comprehensive rock engineering. Vol. 2, 113-171.

ILIFF, J. J., WANG, M., LIAO, Y., PLOGG, B. A., PENG, W., GUNDERSEN, G. A., BENVENISTE, H., VATES, G. E., DEANE, R., GOLDMAN, S. A., NAGELHUS, E. A. \& NEDERGAARD, M. 2012. A Paravascular Pathway Facilitates CSF Flow Through the Brain Parenchyma and the Clearance of Interstitial Solutes, Including Amyloid $\beta$. Science Translational Medicine, 4, 147ra111-147ra111.

KACZMAREK, M., SUBRAMANIAM, R. P. \& NEFF, S. R. 1997. The hydromechanics of hydrocephalus: Steady-state solutions for cylindrical geometry. Bulletin of Mathematical Biology, 59, 295-323.

KHALED, M. Y., BESKOS, D. E. \& AIFANTIS, E. C. 1984. On the theory of consolidation with double porosity-III A finite element formulation. International Journal for Numerical and Analytical Methods in Geomechanics, 8, 101-123.

KHALILI, N. 2003. Coupling effects in double porosity media with deformable matrix. Geophysical Research Letters, 30, n/an/a.

LEVINE, D. N. 1999. The pathogenesis of normal pressure hydrocephalus: a theoretical analysis. Bull Math Biol, 61, 875-916.

LEVINE, D. N. 2000. Ventricular size in pseudotumor cerebri and the theory of impaired CSF absorption. J Neurol Sci, 177, 8594.

LEWALLEN, K. T. \& WANG, H. F. 1998. Consolidation of a double-porosity medium. International Journal of Solids and Structures, 35, 4845-4867.

LI, X., VON HOLST, H. \& KLEIVEN, S. 2013. Influences of brain tissue poroelastic constants on intracranial pressure (ICP) during constant-rate infusion. Comput Methods Biomech Biomed Engin, 16, 1330-43.

MEHRABIAN, A. \& ABOUSLEIMAN, Y. N. 2014. Generalized Biot's theory and Mandel's problem of multiple-porosity and multiple-permeability poroelasticity. Journal of Geophysical Research: Solid Earth, 119, 2745-2763.

MEHRABIAN, A. \& ABOUSLEIMAN, Y. N. 2015. Gassmann equations and the constitutive relations for multiple-porosity and multiple-permeability poroelasticity with applications to oil and gas shale. International Journal for Numerical and Analytical Methods in Geomechanics, 39, 1547-1569.

MIGA, M. I., PAULSEN, K. D., HOOPES, P. J., KENNEDY, F. E., HARTOV, A. \& ROBERTS, D. W. 2000a. In vivo modeling of interstitial pressure in the brain under surgical load using finite elements. J Biomech Eng, 122, 354-63.

MIGA, M. I., PAULSEN, K. D., HOOPES, P. J., KENNEDY, F. E., JR., HARTOV, A. \& ROBERTS, D. W. 2000b. In vivo quantification of a homogeneous brain deformation model for updating preoperative images during surgery. IEEE Trans Biomed Eng, 47, 266-73.

SHOWALTER, R. E. 2000. Diffusion in Poro-Elastic Media. Journal of Mathematical Analysis and Applications, 251, 310-340.

SHOWALTER, R. E. 2002. Diffusion in Deformable Media. In: CHADAM, J., CUNNINGHAM, A., EWING, R. E., ORTOLEVA, P. \& WHEELER, M. F. (eds.) Resource Recovery, Confinement, and Remediation of Environmental Hazards. New York, NY: Springer New York.

SHOWALTER, R. E. \& MOMKEN, B. 2002. Single-phase flow in composite poroelastic media. Mathematical Methods in the Applied Sciences, 25, 115-139.

TULLY, B. \& VENTIKOS, Y. 2010. Cerebral water transport using multiple-network poroelastic theory: application to normal pressure hydrocephalus. Journal of Fluid Mechanics, 667, 188-215.

VARDAKIS, J. C., CHOU, D., TULLY, B. J., HUNG, C. C., LEE, T. H., TSUI, P.-H. \& VENTIKOS, Y. 2016. Investigating cerebral oedema using poroelasticity. Medical Engineering \& Physics, 38, 48-57.

VARDAKIS, J. C., TULLY, B. J. \& VENTIKOS, Y. 2014. Exploring the Efficacy of Endoscopic Ventriculostomy for Hydrocephalus Treatment via a Multicompartmental Poroelastic Model of CSF Transport: A Computational Perspective. PLOS ONE, 8, e84577.

WILSON, R. K. \& AIFANTIS, E. C. 1982. On the theory of consolidation with double porosity. International Journal of Engineering Science, 20, 1009-1035.

WIRTH, B. \& SOBEY, I. 2006. An axisymmetric and fully 3D poroelastic model for the evolution of hydrocephalus. Math Med Biol, 23, 363-88.

ZIENKIEWICZ, O. C. \& SHIOMI, T. 1984. Dynamic behaviour of saturated porous media; The generalized Biot formulation and its numerical solution. International Journal for Numerical and Analytical Methods in Geomechanics, 8, 71-96. 